\title{
Relation between in-vitro Wear and Nanomechanical Properties of Commercial Light-cured Dental Composites Coated with Surface Sealants
}

\author{
Emanuel Santos Jr. ${ }^{\text {a*, Kelma D. P. Nascimento }}{ }^{\text {b }}$, Sérgio S. Camargo Jr. ${ }^{\text {a }}$ \\ ${ }^{a}$ Department of Metallurgical and Materials Engineering, \\ Federal University of Rio de Janeiro - UFRJ, Rio de Janeiro, RJ, Brazil \\ ${ }^{\mathrm{b}}$ Faculty of Dentistry, State University of Rio de Janeiro - UFRJ, Rio de Janeiro, RJ, Brazil
}

Received: December 10, 2012; Revised: April 19, 2013

\begin{abstract}
This work investigates the correlation between the in-vitro wear resistance and the nanomechanical properties of dental sealants commercially available. Mechanical properties, namely hardness $(\mathrm{H})$ and elastic modulus (E), were assessed by nanoindentation technique. The coated samples presented lower $\mathrm{H}$ and $\mathrm{E}$ values than the Z250 composite resin substrate. Such measurements were used to calculate $\mathrm{H} / \mathrm{E}$ ratios. Wear tests were carried out in water by using a pin-on-plate apparatus. Scars formed on the samples were qualitatively examined by optical microscopy, while their wear depths were measured by contact profilometry. Based on the findings, an empirical correlation between the wear depths and $\mathrm{H} / \mathrm{E}$ was obtained. A high $\mathrm{H} / \mathrm{E}$ ratio was associated to surfaces with enhanced wear resistance. For the tribological conditions here employed, the $\mathrm{H} / \mathrm{E}$ ratio could be, therefore, considered a useful parameter for ranking the in-vitro wear of dental sealants.
\end{abstract}

Keywords: light-cured composites, dental sealants, surface sealers, hardness, elastic modulus, nanoindentation, tribology, wear, wear resistance, dentistry, biomaterials

\section{Introduction}

Over the past years, light-cured composites have been widely used in dental restoration. Although relatively easy to handle, cost effective and aesthetically advantageous over other restoration materials, these materials still face some important limitations which hinder their wide-spread use, such as: low wear and fracture resistance, high water absorption and large polymerization shrinkage. Limited color stability, long-term marginal integrity and ability to adhere to the tooth structure are also important factors that must be taken into account when employing such materials. In this way, the use of dental composites under high loads and in large restorations is still avoided. In spite of the many efforts that have been made in order to optimize the mechanical properties of dental composites by adding small inorganic particles into composite resins, wear is still one of the major limiting factors ${ }^{1-6}$. Wear abrasion and attrition generate wear debris, which can be cytotoxic particles or lead to third-body wear mechanisms. Besides to that, biodegradation may reduce the surface hardness and wear resistance due to the presence of microorganisms in the oral cavity ${ }^{7}$.

Surface sealers are currently employed as an attempt to overcome some of these limitations. Generally, dental sealants present low viscosity and surface energy, being able to penetrate, fill in and cover surface defects and, therefore, improve the resin surface by filling the microfissures and small irregularities usually presented in such composites. Further, they improve marginal integrity, reduce microleakage, decrease surface roughness and

*e-mail: emanuel@metalmat.ufrj.br increase wear resistance ${ }^{8-13}$. Beun et al. $(2012)^{14}$ have shown that resin-based pit and fissures sealants commercially available are effective on preventing pit and fissures. On the other hand, if fissures need to be enlarged, flowable resin composites would be more adequate ${ }^{14}$.

Although surface sealants do increase wear resistance of dental composites, the samples filled with surface sealants present reduced surface hardness ${ }^{11}$. This observation is opposite to the common-sense direct correlation between hardness and resistance to abrasive wear. Kim et al. (2002) ${ }^{15}$ have observed a negative correlation between wear and hardness also in case of pit and fissures sealants when studying the effects of different curing methods and storage on their properties ${ }^{15}$. On the other hand, no correlation was found between these two properties in the case of dental composites $^{16}$.

This study is focused on the relation between laboratory wear and mechanical properties of commercial dental sealants. For this purpose, wear tests were carried out in liquid medium using a pin-on-plate equipment, while the mechanical properties were measured by nanoindentation tests. Based on the obtained results, an empirical correlation between wear resistance and mechanical properties of commercial light-cured dental composites coated with surface sealants is established.

\section{Material and Methods}

Acrylic plastic molds of $2.0 \mathrm{~mm}$ thickness with a $5.0 \mathrm{~mm}$ diameter central hole were used to prepare the composite resin samples. The Filtek Z250 ${ }^{\circledR}$ (3M/ESPE, MN, USA) 
Table 1. Summary of the materials data and procedures for applying the surface sealers on the Filtek Z250 ${ }^{\circledR}$ composite resin samples (according to the instructions of the manufacturers).

\begin{tabular}{llllll}
\hline Sample & \multicolumn{1}{c}{ Sealant } & Batch No. & $\begin{array}{c}\text { Air blasting } \\
\text { time (s)* }\end{array}$ & $\begin{array}{c}\text { Photo-curing } \\
\text { time (s) }\end{array}$ & \multicolumn{1}{c}{ Composition } \\
\hline Z250 & none & 9 WM & -- & -- & $\begin{array}{l}\text { Filler: zirconia/silica with size of 0.6 } \mu \text { m; } \\
\text { Organic matrix: Bis-GMA, Bis-EMA, TEGDMA, } \\
\text { and UDMA. }\end{array}$ \\
BC & $\begin{array}{l}\text { BisCover } \\
\text { (Bisco, IL,USA) }\end{array}$ & 0800005064 & -- & 30 & $\begin{array}{l}\text { Ethoxylated Bis-GMA, Urethane acrylate, and } \\
\text { Polyethylene glycol diacrylate. } \\
\text { FG }\end{array}$ \\
$\begin{array}{l}\text { FillGlaze } \\
(\text { Vigodent, RJ, Brazil) }\end{array}$ & $059 / 08$ & 5 & 20 & $\begin{array}{l}\text { Methyl methacrylate, Aliphatic acrylic ester, } \\
\text { Photoinitiator, and Acrylate-epoxy oligomer. }\end{array}$ \\
F & $\begin{array}{l}\text { Fortify } \\
\text { (Bisco, IL,USA) }\end{array}$ & 0700010983 & 5 & 10 & $\begin{array}{l}\text { Urethane Dimethacrylate, and Ethoxylated } \\
\text { Bis-GMA. } \\
\text { Urethane Dimethacrylate, Ethoxylated Bis-GMA, } \\
\text { and Amorphous silica (30\%). }\end{array}$ \\
\hline
\end{tabular}

*perpendicular to the sample surface at $50 \mathrm{~mm}$ far from the substrate (Z250 resin).

microhybrid composite resin was inserted into the mold hole and photo-cured with halogen light for 40 seconds by using the photo-curing Optilux 401 unit (Demetron, TX, USA) with $800 \mathrm{~mW} / \mathrm{cm}^{2}$ light intensity. Then, the Z250 resin samples were abraded with 1200 grit $\mathrm{SiC}$ papers. Afterwards, the samples were soaked in a $37 \% \mathrm{H}_{3} \mathrm{PO}_{4}$ solution for 15 seconds, rinsed with deionized water, and finally, dried in air.

A total of 4 different commercial sealants were employed to fill the composite resin samples, as shown in Table 1 . The sealants were applied following the manufacturers' instructions. A total of six Z250 samples were sealed with each one of the sealants, and six Z250 samples were not sealed. Table 1 summarizes the procedures for each case. All samples were kept in a vial without light and soaked in deionized water for 7 days prior to the experiments. Thicknesses of the applied sealants were measured by contact profilometry with a stylus profilometer Dektak IIA. For each sealant, two samples ( 3 scans on each one) were used to determine the average thickness (total of 6 scans). As shown in Table 2, the thicknesses of the applied sealants varied in the $50-60 \mu \mathrm{m}$ range.

Surface morphologies of the samples were observed by scanning electron microscopy (SEM) with a JEOL 6460LV microscope working at $20 \mathrm{kV}$.

Hardness and elastic modulus of the specimens were measured by nanoindentation tests using a MTS/Agilent G-200 nanoindenter with a Berkovich diamond tip. Indentation measurements were carried out at 16 different spots $30 \mu \mathrm{m}$ apart of each other. The loading-unloading cycle method was employed by applying loads of $1.25,2.5$, and $5.0 \mathrm{mN}$. A standard fused-silica sample with known properties was used for tip area calibration. A Poisson's ratio ( ) of 0.3 was used on calculations $\mathrm{s}^{15}$. The mechanical properties of the samples were calculated using the method developed by Oliver and Pharr ${ }^{17}$.

Wear tests were carried out in liquid medium by using a pin-on-plate apparatus with stationary upper pin and linearly-reciprocating plate. Empress $2^{\circledR}$ (Ivoclar Vivadent, Liechtenstein) $\mathrm{Li}_{2} \mathrm{Si}_{2} \mathrm{O}_{5}$-based ceramic with $2.0 \mathrm{~mm}$ diameter and $7.0 \mathrm{~mm}$ length was used as the pin (counter-face). The pins surfaces were abraded with 600 and 1200 grits SiC
Table 2. Thicknesses of the sealants and wear depths of the scars formed on the surfaces of the composite resin and sealants. Mean value (standard deviation).

\begin{tabular}{|c|c|c|}
\hline Sample & $\begin{array}{c}\text { Thickness } \\
\left(\text { stand. dev.)*( }{ }^{*} \text { m) }\right.\end{array}$ & $\begin{array}{c}\text { Wear depth } \\
\text { (stand. dev. })^{* *}(\mu \mathrm{m})\end{array}$ \\
\hline $\mathrm{Z} 250$ & -- & $10.6(1.7)$ \\
\hline $\mathrm{BC}$ & $58.2(4.5)$ & $3.2(0.9)^{\mathrm{a}}$ \\
\hline FG & $49.9(4.0)$ & $3.0(0.7)^{\mathrm{a}}$ \\
\hline $\mathrm{F}$ & $54.7(5.0)$ & $7.5(2.4)$ \\
\hline FP & $56.9(2.0)$ & $13.6(4.0)$ \\
\hline
\end{tabular}

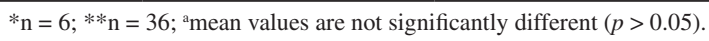

papers before the wear tests. For each wear test, the pin was replaced by a new one. A normal force of $5.0 \mathrm{~N}$, a cycle length of $1.0 \mathrm{~mm}$, linear velocity of $0.5 \mathrm{~mm} / \mathrm{s}$ (frequency of $0.5 \mathrm{~Hz}$ ), and a total number of 10,000 cycles were employed. Such parameters were chosen based on the work of other authors $^{18}$. Deionized water was used as liquid medium at room temperature. A peristaltic pump was used to ensure that the sample and pin were soaked in the liquid during the tests. After the tests, the samples were gently rinsed with deionized water and dried in ambient air. The wear scars formed on the surfaces were observed by optical microscopy. The depths of the wear scars were measured by contact profilometry. A total of 6 measurements in the two directions parallel and perpendicular to the sliding direction were performed on each sample. The results were analyzed using one-way ANOVA followed by 2-tailed Student $t$-test $(\alpha=0.05)$, where $\mathrm{p}<0.05$ indicates a significant difference.

\section{Results and Discussion}

Figure 1 shows the surface morphologies of the asprepared samples observed by scanning electron microscopy (SEM). The composite resin sample (Z250) show a non-uniform surface, containing particles and/or grains irregularly dispersed in the matrix, as expected for a resin composite material. Particle sizes appear to be in the range of a few microns or less. In case of $\mathrm{BC}$ and $\mathrm{F}$ samples, one can observe a very regular, smooth and structureless surface. The FG samples present very similar surface morphologies 

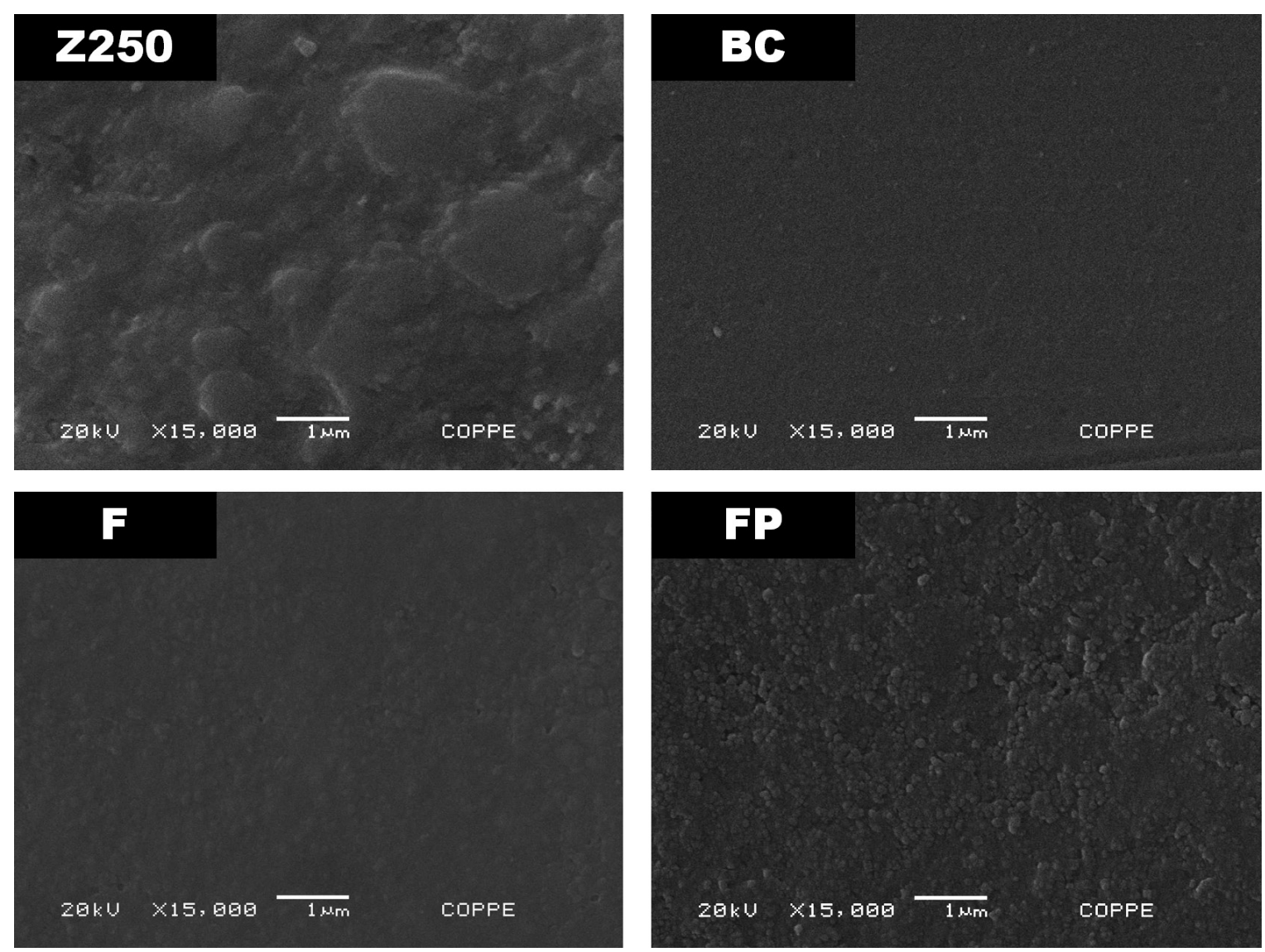

Figure 1. Surface morphologies observed by scanning electron microscopy of the following samples: bare Filtek Z250 ${ }^{\circledR}$ composite resin (Z250), and Z250 samples filled with BisCover ${ }^{\circledR}$ (BC), Fortify ${ }^{\circledR}$ (F) and Fortify Plus ${ }^{\circledR}$ (FP) sealants. The sample filled with FillGlaze ${ }^{\circledR}$ sealant (not shown) presented very similar surface morphology to $\mathrm{F}$ and $\mathrm{BC}$ samples.

to $\mathrm{BC}$ and $\mathrm{F}$ samples and, therefore, are not shown in this figure. FP samples, on the other hand, present slightly irregular surfaces, indicating the presence of particles with sub-micron sizes.

The typical load-displacement curves obtained from the nanoindentation tests are shown in Figure 2 for composite resin (Z250) and BC samples. As one can observe, at similar loads the Z250 sample present much lower penetrations depths compared to the $\mathrm{BC}$ sealant. This means that bare Z250 samples present higher hardness than the samples covered by the BC sealant. The same qualitative result holds for all tested sealants, as will be shown further on. Penetration depths range from about $200 \mathrm{~nm}$ for the hardest samples (Z250) at the lowest loads, to around $1.3 \mu \mathrm{m}$ for the softest samples at the highest loads. Therefore, all results obtained represent the mechanical properties near the surface of the samples at much shallower depths than conventional microhardness measurements.

Figure 3 shows the hardness $(\mathrm{H})$ values as a function of contact depth obtained for the samples at three different applied loads. The hardness obtained for the Z250 samples is around $1.3 \mathrm{GPa}$, regardless of the applied load. The relatively large standard deviations, mainly at the shallow depths, are basically related to the sample surface roughness and the presence of particulate material in the volume bulk (see Figure 1), since contact depths lower than $500 \mathrm{~nm}$ were reached.

In case of the surface sealers, the hardness values range from about $0.1 \mathrm{GPa}$ (FP sample) to approximately $0.8 \mathrm{GPa}$ (F sample), with $\mathrm{BC}$ and FG samples presenting intermediate values of about $0.2-0.3 \mathrm{GPa}$. In all cases, only a slight increase of the hardness values was observed when deeper contact depths were achieved. This small increase is a good indication that the measurements were not influenced by the $\mathrm{Z} 250$ resin substrate. Therefore, the measured values can be related to the sealant materials alone and not to the composite hardness (composite resin + sealant). It is important to stress that this is not necessarily the case of conventional microhardness tests, where much higher loads (in the range of 10 to $100 \mathrm{~g}$ ) may be employed and much deeper depths may be reached, increasing the influence of the resin substrate on the measurements. Nevertheless, the present findings are in qualitative agreement with those from Bertrand et al. ${ }^{11}$ and Catelan et al. ${ }^{19}$, where the application of a surface penetrating sealant reduced the microhardness values of the resin composites.

The obtained elastic modulus (E) values as a function of contact depth for the $\mathrm{Z} 250$ composite resin and the four 


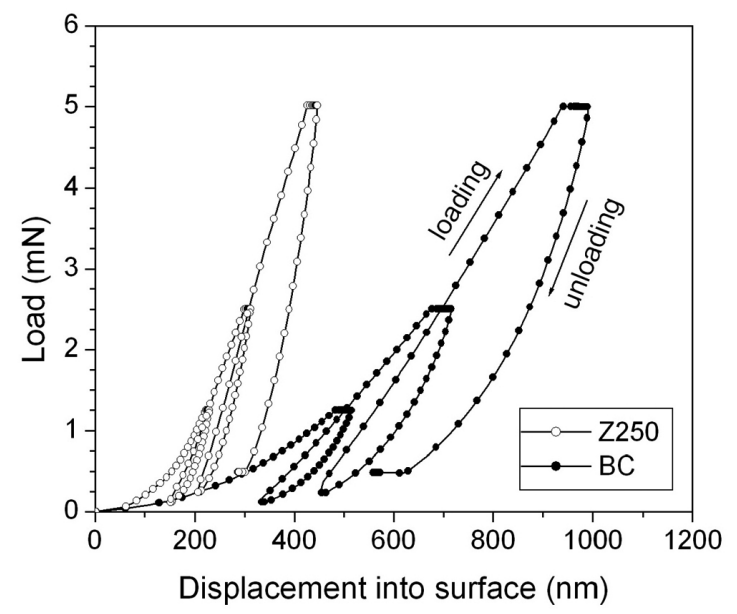

Figure 2. Typical load-displacement curves obtained from the nanoindentation tests for a bare Filtek $\mathrm{Z} 250^{\circledR}$ composite resin (Z250) and the Z250 sample filled with BisCover ${ }^{\circledR}$ (BC) sealant. The samples were submitted loading-unloading cycles with three normal loads: $1.25,2.5$ and $5.0 \mathrm{mN}$.

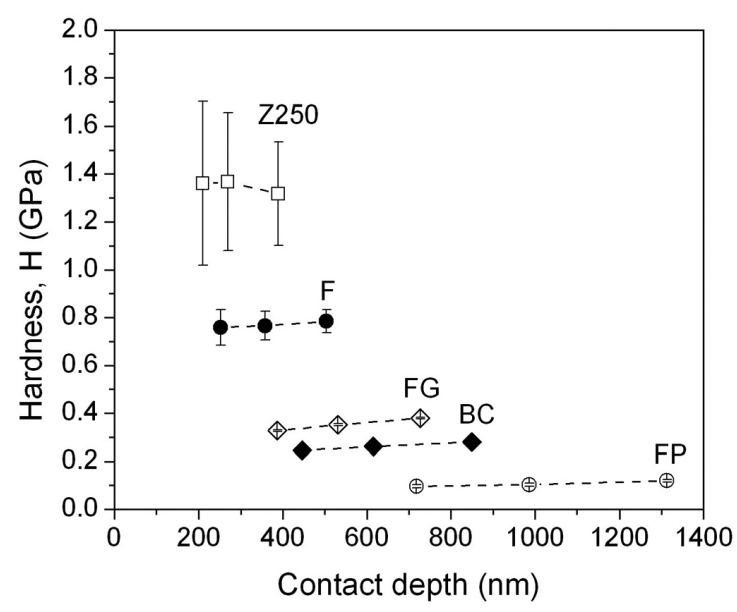

Figure 3. Hardness $(H)$ values obtained from nanoindentation tests as a function of contact depth for the following samples: bare Filtek Z250 ${ }^{\circledR}$ composite resin (Z250), and Z250 samples filled with Fortify Plus $^{\circledR}$ (FP), Fortify ${ }^{\circledR}(\mathrm{F})$, FillGlaze $^{\circledR}(\mathrm{FG})$ and BisCover $^{\circledR}$ (BC) sealants. Dashed lines are guides to the eyes.

sealed samples are presented in Figure 4. The Z250 samples present an elastic modulus of about $22.0 \mathrm{GPa}$, being in good accordance with other authors ${ }^{20}$. Both BC and FP samples present the lowest elastic modulus values around 5.0 GPa, with the FG samples presenting slightly larger values in the 7.0-8.0 GPa range. The F samples present the highest values (around 17.0-18.0 GPa) among the studied surface sealed samples. As in case of the surface hardness, the elastic modulus values of the sealed samples are considerably smaller than those of the bare Z250 composite resin. This finding agrees qualitatively with results for other sealants found in the literature ${ }^{11,20}$.

It is interesting to note that among the tested sealants the only one that incorporates inorganic silica particles in its

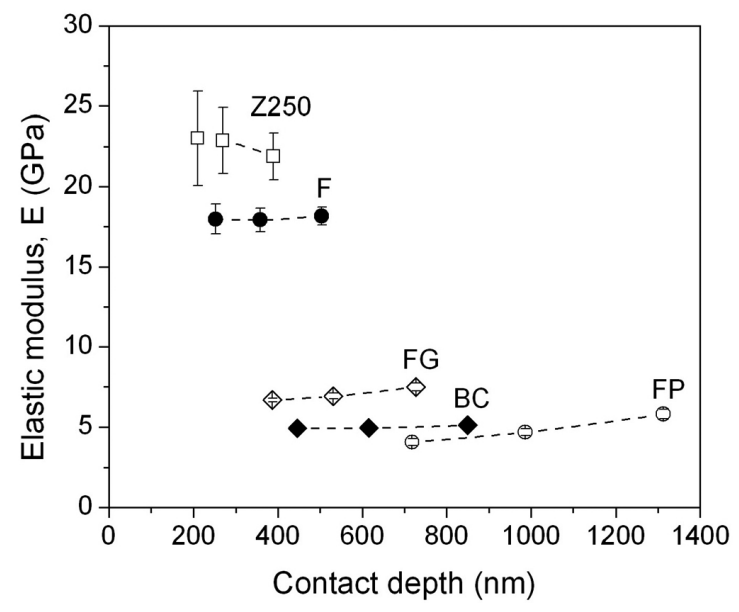

Figure 4. Elastic modulus (E) values obtained from nanoindentation tests as a function of contact depth for the following samples: bare Filtek Z250 ${ }^{\circledR}$ composite resin (Z250), and Z250 samples filled with Fortify Plus $^{\circledR}$ (FP), Fortify ${ }^{\circledR}$ (F), FillGlaze ${ }^{\circledR}$ (FG) and BisCover ${ }^{\circledR}$ (BC) sealants. Dashed lines are guides to the eyes.

composition is the one which presents the smallest values of both hardness and elastic modulus. This is in principle not expected since the purpose of incorporating inorganic particles would be precisely to obtain a composite material with improved mechanical properties. In order to understand this apparently contradictory result, we must first pointed out that one cannot regard the present determination of hardness and elastic modulus by nanoindentation as representative of the (macroscopic) bulk properties of the composite material under service conditions, since a very small volume of the material (with typical dimensions of the order of a micron) is tested during nanoindentation. On the other hand, the volume probed by the present nanoindentation measurements is large enough to include several silica particles, since, as one can see in Figure 1 (FP sample), the particles sizes are well below $1 \mu \mathrm{m}$. Therefore, the conditions employed are in such a way that particle reinforcement should be observed if this mechanism was effective.

In order to evaluate the wear resistance of the commercial sealants, in-vitro wear tests were carried out in liquid medium. The optical microscopy images of the wear scars formed on the surfaces are shown in Figure 5. A qualitative evaluation shows that the FP sealant presented the most damaged surfaces, whereas the FG samples seemed to be the most resistant ones to these tests. In addition, the FG, $\mathrm{BC}$ and $\mathrm{F}$ sealants appeared to be less damaged than the bare $\mathrm{Z} 250$ composite resin. Thus, according to results of these tests, the use of the FG, BC and F surface sealers appear to be effective to reduce wear. On the other hand, the FP samples seemed to be slightly more damaged when compared to the Z250 samples. Such findings were quantitatively confirmed as shown below.

The wear resistance of the samples was quantitatively evaluated by measuring the depths of the wear scars by perfilometry. The depth of each scan was determined and the obtained average values $(n=36)$ are displayed in Table 2 . In agreement with our qualitative evaluation of the wear scars, 

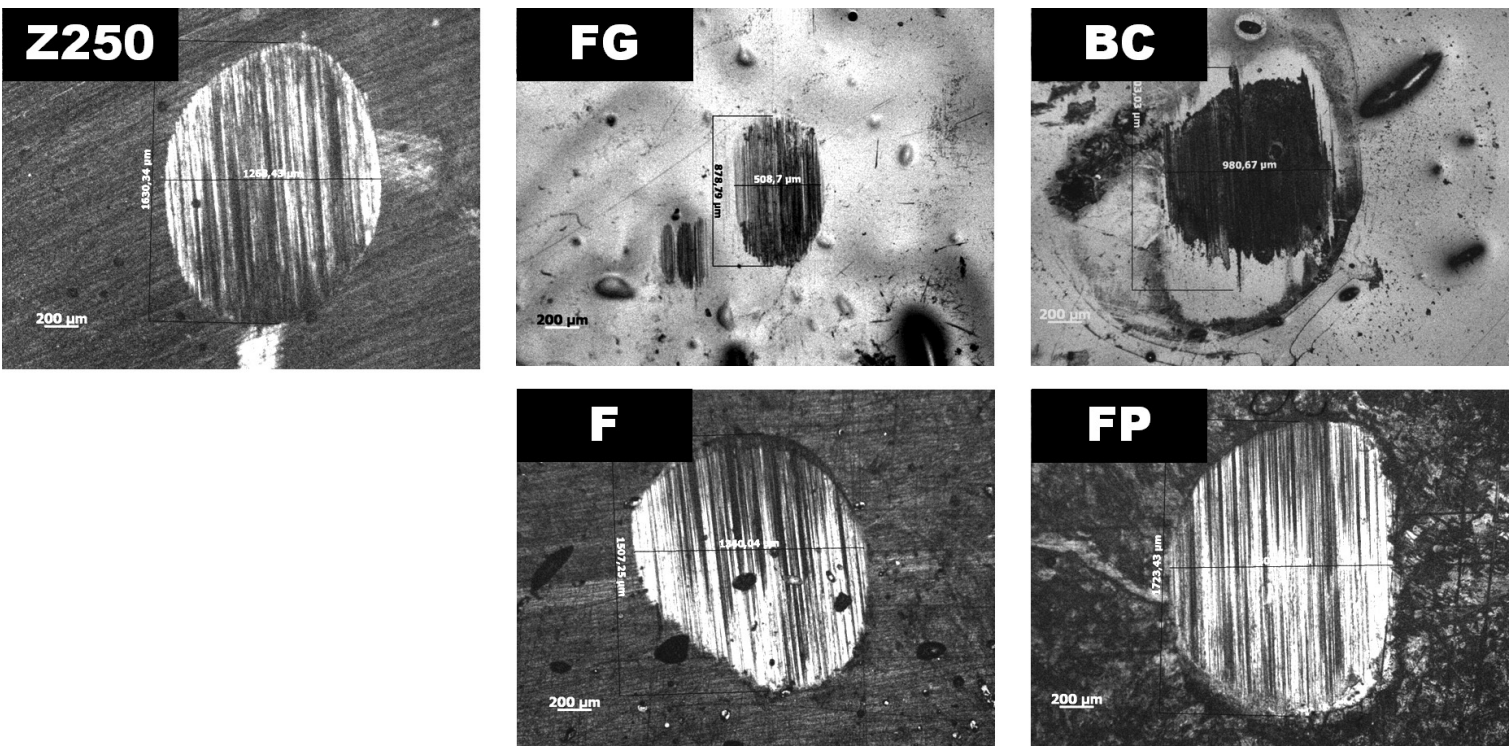

Figure 5. Optical microscopy of the wear scars formed on the following samples: bare Filtek Z250 ${ }^{\circledR}$ composite resin (Z250), and Z250 samples filled with FillGlaze ${ }^{\circledR}$ (FG), BisCover ${ }^{\circledast}$ (BC), Fortify ${ }^{\circledR}$ (F) and Fortify Plus ${ }^{\circledast}$ (FP) sealants.

the BC, FG and F sealants presented smaller wear depths than the Z250 composite resin, whereas the samples coated with FP were more damaged than the bare resin substrate.

When the sealant materials are compared, the BC and FG samples present very similar wear depths, which do not differ significantly $(p>0.05)$. The comparison among the depths of the other three samples (Z250, F and FP) revealed that they are significantly different from each other $(p<0.05)$. Therefore, it can be stated that the BC and FG sealants are the most resistant to wear. In opposition, the FP samples were the most damaged materials. These findings are in accordance to the qualitative results presented in Figure 5. The present results are also in qualitative agreement with in-vivo experiments of other authors which showed that the $\mathrm{F}\left(\right.$ Fortify $^{\circledR}$ ) sealant can improve the wear resistance of composite resins ${ }^{5,9}$. Other in-vitro studies have also reported that both $\mathrm{F}$ and $\mathrm{BC}$ sealants can reduce the wear ${ }^{12,21}$. To certain extent, toothbrushing tests have indicated that surface sealers can ensure the restoration quality, despite having their surfaces roughnesses increased after tests ${ }^{22,23}$.

In the classical theory of wear, hardness is the most important property of a material for defining its wear resistance. The well-known Archard relation, which states an inverse relation between wear rate and hardness, holds for numerous materials under different situations. Therefore, in searching for wear-resistant materials, one generally looks for materials with high hardness. As hard materials usually present high elastic modulus, one can also find a direct correlation between wear resistance and elastic modulus ${ }^{24}$. This is clearly not the case of the present materials, since all investigated sealants present hardness and elastic modulus values smaller than those of the Z250 composite resin, whereas most of them present improved the wear resistance. Furthermore, when comparing the surface sealers among themselves, those materials with intermediate values of both hardness and elastic modulus are exactly those which show the highest wear resistances.

Some polymeric materials (like some elastomers), on the other hand, also present excellent wear resistance although they may be very soft when compared with other type of materials. In this case, however, wear resistance is not determined solely by the hardness of the material. Hill et al. (1997) ${ }^{25}$ have divided the wear rate versus hardness dependence of polyurethane elastomers in three different regions: in the first region (low hardness values) the usual behavior is observed, where wear resistance increases with increasing hardness; in the second region (intermediate hardness values) wear resistance is approximately independent of hardness; finally, in the third region (high hardness values) the wear resistance decreases with increasing hardness ${ }^{25}$. Therefore, in the general case, hardness cannot be the only indicator of the wear resistance polymeric material, as in the present case of the present results.

Some authors have suggested the use of the ratio (H/E) between hardness $\mathrm{H}$ and elastic modulus $\mathrm{E}$ as a measure of the wear resistance of a material or coating, following the original work of Oberle ${ }^{26}$ and Leyland and Matthews ${ }^{27}$. Such parameter combines the desired capability of some types of wear resistant materials to withstand the largest possible elastic deformations (low E) in combination with the smallest permanent deformation (high $\mathrm{H}$ ) under a certain load, which must be sustained without fracture.

Figure 6 presents the H/E ratios derived from the nanoindentation tests for the four sealed samples. Bare $\mathrm{Z} 250$ composite samples were not included in this plot. As one can observe, the $\mathrm{H} / \mathrm{E}$ ratios of both $\mathrm{BC}$ and $\mathrm{FG}$ samples are the highest ones and very similar to each other. The F sample shows a smaller $\mathrm{H} / \mathrm{E}$ ratio, followed by the FP samples which have the smallest values. Some variation of the $\mathrm{H} / \mathrm{E}$ ratio with the applied load was observed, but 
it does not affect the comparison among the four different materials. Assuming a correlation between $\mathrm{H} / \mathrm{E}$ ratio and wear resistance, the results of Figure 6 would indicate that both BC and FG samples are the most wear resistant ones, followed by the F sample, and finally FP sealant, which is exactly the same ranking obtained from the wear tests (see Table 2 and Figure 5).

Figure 7 presents the average values of wear depth as a function of the average values of the obtained $\mathrm{H} / \mathrm{E}$ ratios for the four investigated coated materials. An interesting correlation between the two parameters results, in spite of the large error bars that notably exist in case of the largest wear depths. This is, however, an inherent feature of the wear scars. Based on these results, one can assume that a correlation between $\mathrm{H} / \mathrm{E}$ ratio and wear resistance exists for the case of these sealant materials. Therefore, from the point

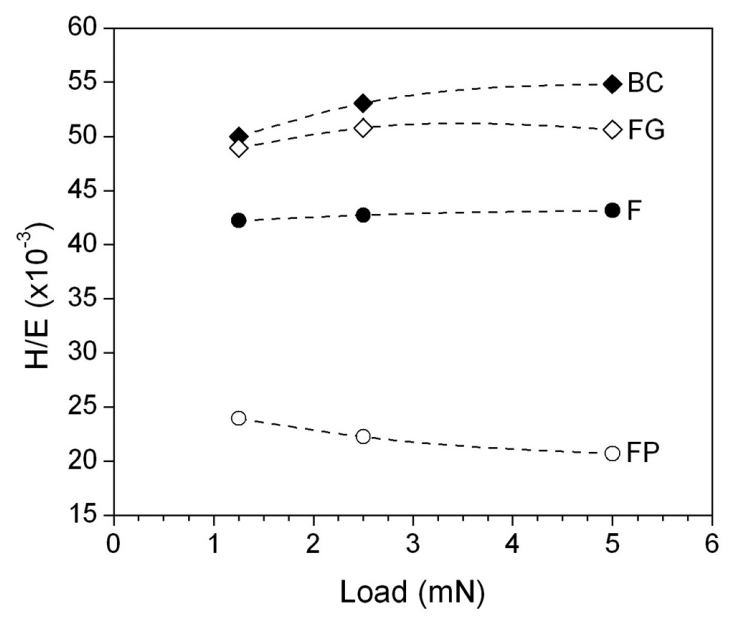

Figure 6. H/E ratios calculated from the nanoindentation tests on the Z250 samples filled with the following sealants: Fortify Plus ${ }^{\circledR}$ (FP), Fortify ${ }^{\circledR}$ (F), FillGlaze ${ }^{\circledR}$ (FG) and BisCover ${ }^{\circledast}$ (BC). Dashed lines are guides to the eyes.

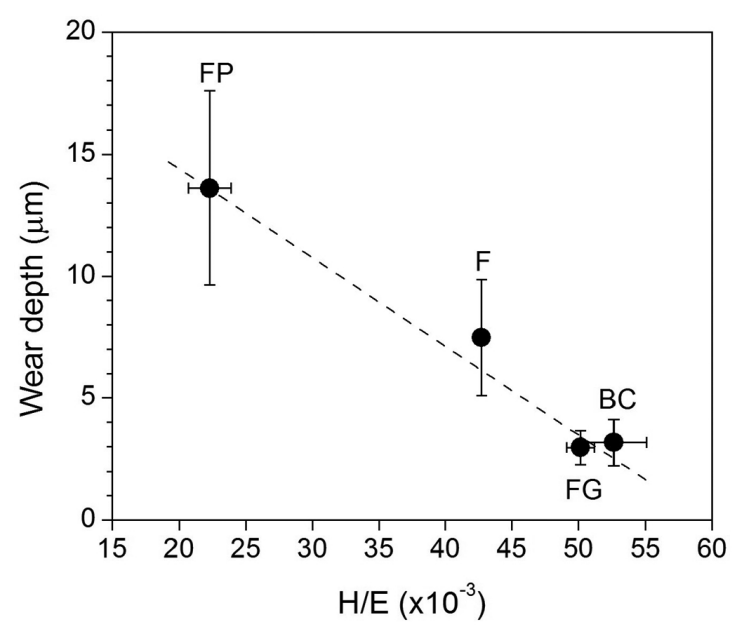

Figure 7. Wear depth as a function of $\mathrm{H} / \mathrm{E}$ ratio for the tested sealants: Fortify Plus ${ }^{\circledR}$ (FP), Fortify ${ }^{\circledR}\left(\right.$ F), FillGlaze ${ }^{\circledR}$ (FG) and BisCover $^{\circledR}(\mathrm{BC})$. Dashed line is a guide to the eyes. of view of wear resistance, the ideal sealant material would be the one with the largest possible $\mathrm{H} / \mathrm{E}$ ratio.

The photo-curing time is a crucial parameter that must be carefully considered regarding the mechanical and wear properties of dental materials. The employment of an accurate photo-curing is critical, since the properties of the surfaces may depend on the energy density, wavelength, exposition time, and distance between the light source and surface $^{15,28}$. As shown by da Silva et al. (2007) ${ }^{29}$, hardness of composite dental resins may also depend on the specific photo-curing unit employed ${ }^{29}$. It would not be surprising if a similar dependence would occur in case of sealant materials. In any case, inadequate photo-curing would affect negatively the properties of the sealant materials, quantitatively changing the obtained results of the materials performance. However, this would affect both the mechanical and wear resistance properties, but should not affect the relation obtained between wear resistance and $\mathrm{H} / \mathrm{E}$ ratio.

It is important to discuss the reasons why the FP samples presented the lowest wear resistance among the investigated sealants. One important point to be considered is that, in contrast to the other sealants, this material presents silica particles in its composition. Therefore, upon analyzing the wear tests results alone, one could eventually attribute the observed noticeable wear damage to the presence of such particles in the wear region, which in principle could contribute to increase abrasion during the experiment, thus increasing the wear of such samples. Regarding the experimental conditions here employed, with a continuous flow of liquid media during the measurements, this not seems to be the case.

Yang et al. $(1991)^{30}$ have investigated the abrasive wear of silicone rubbers filled with inorganic particles and found that the detailed interaction between the filler particles and the hard abrasive asperities is the key to determine the wear behavior $^{30,31}$. The detailed wear mechanism is determined by the relation between the filler particle dimensions and the asperities spacing ${ }^{30,31}$. When the particle dimensions are larger than the asperities spacing a load-supporting action takes place and wear of the filler particles dominates. When particle dimensions and asperities spacing are similar, the load-supporting action decreases and filler pull-out can occur resulting in much higher wear rates. Finally, when the particle dimensions are small compared with asperities spacing, there is no load-supporting action and the wear behavior resemble that of the homogeneous material. In the last two cases, good adhesion of filler particles to the matrix is of fundamental importance in order to improve wear resistance.

Microscopic examination of FP samples showed that particles sizes are in the submicron range and, therefore, we can expect our wear tests to be in the regime where particle sizes are smaller (or maybe similar) to the asperities spacing and conclude that adhesion of particles to the polymeric matrix is very important in this case. The low values obtained for the mechanical properties $\mathrm{H}$ and $\mathrm{E}$ (and also $\mathrm{H} / \mathrm{E}$ ) suggest that very small reinforcement (if any) is provided by the filler particles in case of these samples. Therefore, it seems likely that only the properties of the sample matrix are being probed in the present experiments. In this way 
it is not surprising that the results of FP sample fall in the same wear versus $\mathrm{H} / \mathrm{E}$ relation as the other coated samples.

As a matter of fact, a straight correlation between results from clinical trials and laboratory wear tests is not trivial. In fact, many efforts have been done to correlate the wear results originated from in-vitro tests and those obtained from in-vivo studies. Recently, Heintze et al. (2012) ${ }^{32}$ compared the in-vivo wear to six different laboratory wear tests $^{32}$. Accordingly, none of the tested methods were able to predict the clinical wear of conventional dental resins ${ }^{32}$. In this case, the in-vitro wear results are limited to the fact that they do not reflect the clinical wear observed. The oral environment involves, indeed, many other issues that affect the wear of dental resins, for example: $\mathrm{pH}$, corrosion, time, forces, movement directions, etc. In practice, one can hardly mimic all the conditions in simple laboratory tests. Notwithstanding, the in-vitro wear methods can be useful to evaluate and rank the materials performance before clinical trials, and also to study their wear mechanisms under certain (or limited) conditions. The latter depends on the laboratory method used, since different mechanisms can be resulted from different methods for the same material ${ }^{33}$. In case of pin-on-plate (reciprocating) wear tests, adhesive, abrasive and/or fatigue are the main wear mechanisms presented. Abrasive wear takes places due to the interposition of wear debris between the resin and antagonist pin, thereby generating the three-body wear. Afterwards, fatigue wear

\section{References}

1. Leinfelder KF. Posterior composite resins - the materials and their clinical performance. Journal of the American Dental Association. 1995; 126:663-664. PMid:7759692.

2. Manhart J, Kunzelmann KH, Chen HY and Hickel R. Mechanical properties and wear behavior of light-cured packed composite resins. Dental Materials. 2000; 16:33-40. http:// dx.doi.org/10.1016/S0109-5641(99)00082-2

3. Zantner C, Kielbassa AM, Martus P and Kunzelmann KH. Sliding wear of 19 commercially available composites and compomers. Dental Materials. 2004; 20:277-285. http://dx.doi. org/10.1016/S0109-5641(03)00104-0

4. Nagarajan VS, Jahanmir S and Thompson VP. In vitro wear contact of dental composites. Dental Materials. 2004; 20:6371. http://dx.doi.org/10.1016/S0109-5641(03)00069-1

5. Blalock JS, Chan DCN, Browning WD, Callan R and Hackman S. Measurement of clinical wear of two packable composites after 6 months in service. Journal of Oral Rehabilitation. 2006; 33:59-63. PMid:16409518. http://dx.doi. org/10.1111/j.1365-2842.2006.01540.x

6. Tan YN, Liu Y, Grover LM and Huang B. Wear behavior of light-cured dental composites filled with porous glass-ceramic particles. Journal of Mechanical Behavior of Biomedical Materials. 2010; 3:77-84. PMid:19878904. http://dx.doi. org/10.1016/j.jmbbm.2009.04.004

7. Bettencourt AF, Neves CB, De Almeida MS, Pinheiro LM, Oliveira SA, Lopes LP et al. Biodegradation of acrylic based resins: A review. Dental Materials. 2010; 26:e171-e180. PMid:20189238. http://dx.doi.org/10.1016/j. dental.2010.01.006 can lead to micron-cracks formation and propagation (not seen in our samples), depending on the forces applied and number of cycles. Hence, the found $\mathrm{H} / \mathrm{E}$ relation could be applied to dental sealants when the wear mechanisms under consideration resemble somehow those presented for pinon-plate tests.

\section{Conclusions}

The present results showed that the wear resistance of a commercial microhybrid resin composite can be enhanced by the use of surface sealers. Both hardness and elastic modulus of the sealants were found to be smaller than those of the composite resin. The wear resistance of the surface sealers was associated with their $\mathrm{H} / \mathrm{E}$ ratios. Regarding the tribological conditions here employed, the relation $\mathrm{H} / \mathrm{E}$ could be also used to rank the laboratory wear performance of such materials. From the point of view of wear resistance, the desired properties of a sealant is withstand the largest possible elastic deformations (low E) in combination with the smallest permanent deformation (high $\mathrm{H}$ ) under a certain load, which must be sustained without fracture.

\section{Acknowledgements}

This work was partially supported by CAPES and CNPq Brazilian funding agencies.

8. Dickinson GL, Leinfelder KF, Mazer RB and Russell CM. Effect of surface penetrating sealant on wear rate of posterior composite resins. Journal of the American Dental Association. 1990; 121:251-255. PMid:2144862.

9. Dickinson GL and Leinfelder KF. Assessing the long-term effect of a surface penetrating sealant. Journal of the American Dental Association. 1993; 124:68-72. PMid:8335801.

10. Kawai K and Leinfelder KF. Effect of surface-pentrating sealant on composite wear. Dental Materials. 1993; 9:108-113. http:// dx.doi.org/10.1016/0109-5641(93)90085-5

11. Bertrand MF, Leforestier E, Muller M, Lupi-Pégurier L and Bolla M. Effect of surface penetrating sealant on surface texture and microhardness of composite resins. Journal of Biomedical Materials Research (Applied Biomaterials). 2000; 53:658-663. http://dx.doi.org/10.1002/1097-4636(2000)53:6<658::AIDJBM7>3.0.CO;2-O

12. Prakki A, Ribeiro IWJ, Cilli R and Mondelli RF. Assessing the tooh-restoration interface wear resistance of two cementation techniques: effect of a surface sealant. Operative Dentistry. 2005; 30:739-746. PMid:16382597.

13. Owens BM and Johnson WW. Effect of the new generation surface sealants on the marginal permeability of class $\mathrm{V}$ resin composite restorations. Operative Dentistry. 2006; 31:481-488. PMid:16924989. http://dx.doi.org/10.2341/05-77

14. Beun S, Bailly C, Devaux J and Leloup G. Physical, mechanical and rheological characterization of resin-based pit and fissure sealants compared to flowable resin composites. Dental Materials. 2012; 28:349-359. PMid:22119547. http://dx.doi. org/10.1016/j.dental.2011.11.001

15. Kim JW, Jang KT, Lee SH, Kim CC, Hahn SH and GarcíaGodoy F. Effect of curing method and curing time on the 
microhardness and wear of pit and fissure sealants. Dental Materials. 2002; 18:120-127. http://dx.doi.org/10.1016/S01095641(01)00030-6

16. Yap AUJ, Ong LFKL, Teoh SH and Hastings GW. Comparative wear ranking of dental restoratives with the BIOMAT wear simulator. Journal of Oral Rehabilitation. 1999; 26:228235. PMid:10194732. http://dx.doi.org/10.1046/j.13652842.1999.00359.x

17. Oliver WC and Pharr GM. An improved technique for determining hardness and elastic-modulus using load and displacement sensing indentation experiments. Journal of Materials Research. 1992; 7:1564-1583. http://dx.doi. org/10.1557/JMR.1992.1564

18. Ramalho A and Antunes PV. Reciprocating wear test of dental composites against human teeth and glass. Wear. 2007; 263:1095-1104. http://dx.doi.org/10.1016/j. wear.2007.01.086

19. Catelan A, Briso ALF, Sundfeld RH and Dos Santos PH. Effect of artificial aging on the roughness and microhardness of sealed composites. Journal of Esthetic Restorative Denistry. 2010; 22:324-330. PMid:21029336. http://dx.doi. org/10.1111/j.1708-8240.2010.00360.x

20. Pongprueksa P, Kuphasuk W and Senawonyse. The elastic moduli across various types of resin/dentin interfaces. Dental Materials. 2008; 24:1102-1106. PMid:18304626. http://dx.doi. org/10.1016/j.dental.2007.12.008

21. Shinkai K, Suzuki S, Leinfelder KF and Katoh Y. Effect of surface penetrating sealant on wear resistance of luting agents. Quintessence International. 1994; 25:767-771. PMid:7568681.

22. Dos Santos PH, Pavan S, Suzuki TYU, Briso AL, Assunção WG, Sinhoreti MA et al. Effect of fluid resins on the surface roughness andtopography of resin composite restorations analyzed by atomic force microscope. Journal of the Mechanical Behavior of Biomedical Materials. 2011; 4:433-439. PMid:21316631. http://dx.doi.org/10.1016/j.jmbbm.2010.12.004

23. Zimmerli B, Koch T, Flury S and Lussi A. The influence of toothbrushing and coffee staining on different composite surface coatings. Clinical Oral Investigation. 2012; 16:469-
479. PMid:21331636. http://dx.doi.org/10.1007/s00784-0110522-2

24. Bhushan B. Introduction to Tribology. New York: John Wiley \& Sons; 2002.

25. Hill DJT, Killeen MI, O’Donnell JH, Pomery PJ, St John D and Whittaker AK. Laboratory wear testing of polyurethane elastomers. Wear. 1997; 208:155-160. http:// dx.doi.org/10.1016/S0043-1648(96)07514-X

26. Oberle TL. Properties influencing the wear of metals. Journal of Metals. 1951; 3:438-439.

27. Leyland A and Matthews A. On the significance of the H/E ratio in wear control: a nanocomposite coating approach to optimised tribological behaviour. Wear. 2000; 246:1-11. http:// dx.doi.org/10.1016/S0043-1648(00)00488-9

28. Rueggeberg FA. State-of-the-art: dental photocuring - A review. Dental Materials. 2011; 27:39-52. PMid:21122903. http:// dx.doi.org/10.1016/j.dental.2010.10.021

29. Silva GR, Simamoto PC Jr, Da Mota AS and Soares CJ. Mechanical properties of light-curing composites polymerized with different laboratory photo-curing units. Dental Materials Journal. 2007; 26:217-223. PMid:17621937. http://dx.doi. org/10.4012/dmj.26.217

30. Yang ACM, Ayala JE, Bell A and Scott IC. Effect of filler particles on abrasive wear of elastomer-based composites. Wear. 1991; 146:349-366. http://dx.doi.org/10.1016/00431648(91)90074-5

31. Yang ACM, Ayala JE and Scott JC. Abrasive wear in filled elastomers. Journal of Materials Science. 1991; 26:5823-5837. http://dx.doi.org/10.1007/BF01130121

32. Heintze SD, Faouzi M, Rousson V and Ozcan M. Correlation of wear in vivo and six laboratory wear methods. Dental Materials. 2012; 28:961-973. PMid:22698644. http://dx.doi. org/10.1016/j.dental.2012.04.006

33. Heintze SD, Zappini G and Rousson V. Wear of ten dental restorative materials in five wear simulators - Results of a round robin test. Dental Materials. 2005; 21:304-317. PMid:15766577. http://dx.doi.org/10.1016/j.dental.2004.05.003 